Research Article

\title{
Prostate-Specific Antigen Kinetics Effects on Outcomes of Low-Volume Metastatic Prostate Cancer Patients Receiving Androgen Deprivation Therapy
}

\author{
Yen-Chi Lin ${ }^{1},{ }^{1}$ Po-Hung Lin, ${ }^{1}$ I-Hung Shao, ${ }^{1}$ Yuan-Cheng Chu, ${ }^{1}$ Hung-Cheng Kan, ${ }^{1}$ \\ Chung-Yi Liu, ${ }^{2}$ Kai-Jie Yu, ${ }^{1}$ Ying-Hsu Chang, ${ }^{2}$ See-Tong Pang, ${ }^{1}$ Jhen-Ling Huang, ${ }^{3}$ \\ and Cheng-Keng Chuang $\mathbb{D}^{1}$ \\ ${ }^{1}$ Division of Urology, Department of Surgery, Chang Gung Memorial Hospital and Chang Gung University, Taoyuan, Taiwan
${ }^{2}$ Division of Urology, Department of Surgery, New Taipei Municipal TuCheng Hospital,
Chang Gung Memorial Hospital and Chang Gung University, New Taipei City, Taiwan
${ }^{3}$ Center for Big Data Analytics and Statistics, Chang Gung Memorial Hospital, Taoyuan, Taiwan
}

Correspondence should be addressed to Cheng-Keng Chuang; ckchuang@gmail.com

Received 9 June 2021; Accepted 14 August 2021; Published 27 August 2021

Academic Editor: Nihal Ahmad

Copyright (c) 2021 Yen-Chi Lin et al. This is an open access article distributed under the Creative Commons Attribution License, which permits unrestricted use, distribution, and reproduction in any medium, provided the original work is properly cited.

Background. The present study aimed to analyse factors influencing the effects of androgen deprivation therapy (ADT) in patients with newly diagnosed metastatic castration-naïve prostate cancer (mCNPC), especially in low-volume disease (LVD), according to subclassification of metastatic prostate cancer established by the CHAARTED trial. Materials and Methods. We reviewed 648 patients with newly diagnosed mCNPC receiving ADT at Chang Gung Memorial Hospital from January 2007 to December 2016. Basic characteristics and PSA kinetics profile were subsequently evaluated. Results. $48.3 \%$ of LVD patients progressed to castration-resistant prostate cancer (mCRPC). Among them, CRPC group had significantly shorter time to PSA nadir (TTN) and faster time from PSA nadir to CRPC (TFNTC) $(p<0.001)$ compared to non-CRPC group. PSA doubling time $($ PSADT) $<4$ months tended to be associated with faster disease progression and shorter overall survival (OS). Among all patients with metastatic prostate cancer, those with shorter TTN $<9$ months, higher nadir PSA level $\geq 1 \mathrm{ng} / \mathrm{mL}$, and shorter PSADT $<3$ months had increased tendency for biochemical progression. Conclusions. PSADT is an effective clinical predictor for disease progression and survival in LVD. Other PSA kinetics including TTN and TFNTC, though not the major predictors for disease progression or OS in LVD, might be the predictors for disease control status.

\section{Background}

Prostate cancer is one of the most common malignancies worldwide and the fifth most common cancer in Taiwan, with an age-standardised prostate cancer rate of 31.65 per 100,000 individuals in 2017 and metastatic prostate cancer (mPCa) accounting for nearly $30 \%$ of new cases [1-4]. Androgen deprivation therapy (ADT) has been the gold standard for patients with $\mathrm{mPCa}$. Unfortunately, after receiving $\mathrm{ADT}$, most of the prostate cancer cells develop drug resistance and progress to castration-resistant prostate cancer (CRPC), necessitating chemotherapy or second-line hormone therapy if feasible. Recent clinical trials and research have shown that upfront chemotherapy plus ADT promoted significantly longer overall survival (OS) in high-volume disease (HVD) [5-8]. "Tumor volume," a new subclassification of metastatic prostate cancer established by the CHAARTED trial, can be classified as "high-volume" (visceral metastases and/or four or more bone metastases with at least one outside the vertebral column and pelvis) or "low-volume." Moreover, this study showed that patients with HVD receiving a combination of ADT and chemotherapy had a longer median OS (49.2 months) than 
those receiving ADT alone (32.2 months), but LVD did not have survival benefit receiving chemohormonal therapy [5].

Apart from tumor volume, prostate-specific antigen (PSA) has been recognised as an important biomarker for predicting treatment response and disease progression in prostate cancer during ADT. The PSA kinetics profile, including initial PSA level (iPSA) [9], time to PSA nadir (TTN) [9-12], nadir PSA level $[9,13,14]$, PSA decline pattern [15-17], and PSA doubling time (PSADT) [18], had been shown to reflect tumor burden and predict outcomes in patients with prostate cancer under ADT. Other than PSA level and related factors, pretreatment parameters, including Gleason grade group, haemoglobin $(\mathrm{Hb})$, and alkaline phosphatase (ALP), have also been identified as prognostic or predictive biomarkers in mCNPC patients [19].

Most previous studies had included populations comprising patients with metastatic castration-naivve prostate cancer (mCNPC) or metastatic castration-resistant prostate cancer (mCRPC). However, since the CHAARTED trial, the concept of tumor volume had been integrated into the management of metastatic prostate cancer. As such, identifying reliable predicting factors for disease progression or overall survival according to different tumor volumes during ADT would be helpful in the prompt formulation of better treatment strategies for patients with poor prognostic characteristics. Several studies have investigated factors influencing disease burden in HVD $[19,20]$, while few of them discussed the risk factors for disease burden in lowvolume disease (LVD). Thus, the current study aimed to explore the risk factors, particularly PSA kinetics, associated with poor prognosis, including shorter OS and shorter time to CRPC (the duration from initiation of ADT to biochemical CRPC status) in LVD under ADT.

\section{Materials and Methods}

2.1. Study Design and Patient Selection. We retrospectively evaluated patients with newly diagnosed metastatic prostate cancer between January 2007 and December 2016 who had received primary ADT (either surgical or medical castration, with or without antiandrogen) at Chang Gung Memorial Hospital (CGMH) Linkou branch. Patients were grouped into HVD and LVD according to the CHAARTED trial. This study adhered to the tenets of the Declaration of Helsinki and was approved by the Institutional Review Board (IRB) of Chang Gung Medical Foundation (IRB number 201801377B0). Patient consent is not required for observational studies.

2.2. Clinical Data and Outcome Collection. CRPC was diagnosed based on biochemical progression (three consecutive spikes in PSA 1 week apart, of which two were $50 \%$ higher than the nadir, and PSA $>2 \mathrm{ng} / \mathrm{mL}$ ) according to the European Association of Urology guideline. OS was defined as the period from diagnosis until death by any cause. Baseline patient demographics and posttreatment characteristics, including age at diagnosis, clinical $\mathrm{M}$ staging, iPSA
(PSA level upon diagnosis), Gleason grade group (according to the classification of International Society of Urological Pathology [21]: grade group 1, Gleason score $\leq 6$; grade group 2, Gleason score $3+4=7$; grade group 3, Gleason score 4+3=7; grade group 4, Gleason score 8; and grade group 5, Gleason score $\geq 9)$, initial haemoglobin $(\mathrm{Hb})$, calcium (Ca), and alkaline phosphatase (ALP) level, were determined. PSA kinetics profiles were defined based on previous related studies [11, 19, 22, 23], including TTN (defined as the duration from ADT initiation to PSA nadir), nadir PSA level, 3 months' PSA reduction rate (defined as (iPSA, posttreatment 3 months' PSA)/iPSA), PSA reduction rate (PSARR) (defined as $100 \times(\mathrm{iPSA}-\mathrm{PSA}$ nadir/iPSA)/ TTN) [24], time to CRPC (the duration from ADT initiation to biochemical CRPC status), time from PSA nadir to CRPC (TFNTC) (the duration from PSA nadir to CRPC status), and PSADT (defined as $\log 2 \times$ (time interval)/log (PSA value)-log (nadir PSA)). Patients with insufficient imaging reports for determining volume status or excessive missing data were excluded.

Previous studies have demonstrated that several cutoff points for PSA-related factors, including iPSA, TTN, PSA nadir, and PSADT, predicted disease progression or OS $[10,11,13,24,25]$. Moreover, some studies have utilised the receiver operating characteristic curve to determine cutoff points, while others use median values. The current study chose median values as the optimal cutoff points for the different parameters.

2.3. Statistical Analysis. All statistical analyses were performed using SAS software version 9.4 (SAS Institute Inc., Cary, NC). Nominal variables are presented as means and standard deviations, while nonnominal variables are presented as medians and interquartile ranges. The Chi-square test was used to compare categorical variables, while the independent $t$-test was used to compare continuous variables. OS and CPRC-free survival were evaluated using the Kaplan-Meier method. The multivariate Cox proportionalhazards model was used to determine the association between risk factors and OS or CPRC-free survival. All $p$ values reported were two sided with $p<0.05$ indicating statistical significance.

\section{Results}

3.1. Characteristics of the Study Population. A total of 918 patients with newly diagnosed metastatic prostate cancer at CGMH from January 2007 to December 2016 were identified. After excluding those who had received either chemotherapy or radiotherapy and those with incomplete information, a total of 648 patients receiving primary ADT were ultimately analysed. In the study population (Table 1), included patients had a median age of 75 (IQR 68-80) years, with $352(54.3 \%)$ classified as HVD and 296 (45.7\%) as LVD according to the CHAARTED trial. At the median follow-up of 34 months (range from 1 month to 137 months), a total of $371(57 \%)$ patients died during the study period. The median OS was 48 months (Table 2), while those with HVD had a 
TABLE 1: Baseline characteristics.

\begin{tabular}{|c|c|c|c|}
\hline Patient demographics & $\operatorname{HVD}(n=352,54.3 \%)$ & LVD $(n=296,45.7 \%)$ & $p$ value \\
\hline Age, median, year (IQR) & $75(68-80)$ & $74(68-80)$ & 0.2242 \\
\hline M stage, $n(\%)$ & & & $<0.0001$ \\
\hline $1 \mathrm{~A}$ & 0 & $72(24.3 \%)$ & \\
\hline $1 \mathrm{~B}$ & $268(76.1 \%)$ & $224(75.7 \%)$ & \\
\hline $1 \mathrm{C}$ & $84(23.9 \%)$ & 0 & \\
\hline Gleason grade group, $n(\%)$ & & & 0.0013 \\
\hline 1 & 0 & $8(3.3 \%)$ & \\
\hline 2 & $6(2.1 \%)$ & $12(4.9 \%)$ & \\
\hline 3 & $26(9.1 \%)$ & $34(13.9 \%)$ & \\
\hline 4 & $64(22.3 \%)$ & $47(19 \%)$ & \\
\hline 5 & $191(66.5 \%)$ & $145(58.9 \%)$ & \\
\hline Initial PSA level, ng/ml, median (IQR) & $511.5(181.25-1234.47)$ & $98.2(45-256.6)$ & $<0.0001$ \\
\hline $\mathrm{Hb}, \mathrm{g} / \mathrm{dL}$, median (IQR) & $11.5(9.7-13.2)$ & $12.7(11.6-13.9)$ & $<0.0001$ \\
\hline $\mathrm{Ca}, \mathrm{g} / \mathrm{dL}$, median (IQR) & $8.7(8.2-9.1)$ & $8.8(8.3-9.1)$ & 0.9528 \\
\hline ALP, U/L, median (IQR) & $146(94-324)$ & $74(60-101)$ & $<0.0001$ \\
\hline CRPC, $n(\%)$ & & & $<0.0001$ \\
\hline Yes & $232(65.9 \%)$ & $143(48.3 \%)$ & \\
\hline No & $120(34.1 \%)$ & $153(51.7 \%)$ & \\
\hline \multicolumn{4}{|l|}{ PSA kinetics after ADT } \\
\hline TTN, month, median (IQR) & $7.15(3.7-13.3)$ & $11.9(6.25-20.9)$ & $<0.0001$ \\
\hline Nadir PSA level, ng/ml, median (IQR) & $2.2(0.23-17.18)$ & $0.23(0.02-1.7)$ & 0.0015 \\
\hline PSARR, \%/month, median (IQR) & $12.5(6.8-22.04)$ & $7.99(4.38-14.9)$ & 0.0002 \\
\hline TFNTC, month, median (IQR) & $3.25(2.5-7.9)$ & $8.3(2.7-26.1)$ & $<0.0001$ \\
\hline PSADT, month, median (IQR) & $2.3(1.4-4.15)$ & $3.6(1.9-7)$ & $<0.0001$ \\
\hline
\end{tabular}

CRPC: castration-resistant prostate cancer, TTN: time to PSA nadir, PSARR: PSA reduction rate, TFNTC: time from PSA nadir to CRPC, and PSADT: PSA doubling time.

significantly shorter median OS than those with LVD (35 vs. 72 months; $p<0.0001$ ) (Figure 1(a)).

\subsection{Comparison between High-Volume Disease and Low-} Volume Disease. A total of 375 (57.9\%) patients progressed to biochemical CRPC status (Table 2); among them, 232 (61.9\%) patients were HVD and 143 (38.1\%) were LVD. The median time to CRPC in all patients, those with HVD, and those with LVD was $16.5,13$, and 26 months, respectively $(p<0.0001)$ (Figure 1(b)). Significant differences in baseline characteristics and PSA kinetics, including $\mathrm{Hb}$, ALP, iPSA, Gleason grade group, TTN, PSARR, PSA nadir level, TFNTC, and PSADT, were observed between patients with HVD and LVD (Table 1). In the subgroup analysis, patients with LVD were divided into CRPC group $(48.3 \%)$ and nonCRPC group (51.7\%). Among them, CRPC group had similar nadir PSA level, 3-month PSA reduction rate, and PSADT, but significantly shorter TTN (8.7 vs. 16.7 months) and faster TFNTC (4.3 vs. 15.6 months) $(p<0.001)$ compared to the non-CRPC group (Table 3).

\subsection{Affecting Factors for Overall Survival and Disease} Progression. Multivariate analysis of all included patients (mCNPC) revealed that those with TTN $<9$ months, nadir PSA level $\geq 1 \mathrm{ng} / \mathrm{mL}$, and PSADT $<3$ months had increased tendency for biochemical progression (Table 4 ), while those with TTN $<9$ months, nadir PSA level $\geq 1 \mathrm{ng} / \mathrm{mL}$, duration of PSA nadir $<5$ months, PSADT $<3$ months, and time to CRPC $<17$ months had increased risk for shorter OS (Table 5). Univariate analysis of patients with LVD showed that
Gleason grade group and PSA kinetics including iPSA, PSARR, TFNTC, TTN, and PSADT were statistically significant parameters for disease progression and OS; however, multivariate analysis of patients with LVD showed that only PSADT ( $<4$ months) was associated with increased risk for early disease progression to CRPC and shorter OS (Tables 6 and 7).

\section{Discussion}

Research has shown that upfront chemotherapy with firstline ADT significantly improved OS in patients with highvolume mCNPC [5]. Accordingly, previous studies had identified age, ECOG performance status, Gleason grade group, pretreatment $\mathrm{Hb}, \mathrm{ALP}, \mathrm{LDH}$, nadir PSA level, TTN, and PSADT as prognostic factors for mPCa $[9,19,22]$. Apart from the aforementioned factors, Guangjie et al. demonstrated that patients who exhibited a rapid decrease in PSA levels during the initial ADT phase were at increased risk for progression to CRPC [16]. Nakayama et al. introduced the concept that PSA kinetics and early PSA decline were associated with time to PSA progression differently in patients with $\mathrm{mCRPC}$ receiving abiraterone acetate. However, the aforementioned study had a limited number of patients and events [26]. Accordingly, the present study found that PSA kinetics was strongly associated with either risk for disease progression or OS. To date, three major clinical trials, namely, GETUG-AFU 15, CHAARTED, and STAMPEDE, had incorporated early chemohormonal therapy into their treatment stagey for mCNPC [27]. Among the aforementioned trials, only CHAARTED had classified the study 
TABLE 2: Overall baseline characteristics.

\begin{tabular}{lc}
\hline Patient demographics & All patients $(n=648)$ \\
\hline Age, median, year (IQR) & $75(68-80)$ \\
Overall survival, median, month (IQR) & $48(24-106)$ \\
Time to CRPC, median, month (IQR) & $16.5(8-36)$ \\
M stage, $n$ (\%) & \\
1A & $72(11.11 \%)$ \\
1B & $492(75.93 \%)$ \\
1C & $84(12.96 \%)$ \\
Gleason grade group, $n$ (\%) & \\
1 & $8(1.5 \%)$ \\
2 & $18(3.38 \%)$ \\
3 & $60(11.26 \%)$ \\
4 & $111(20.83 \%)$ \\
5 & $336(63.04 \%)$ \\
Missing & 115 \\
Initial PSA level, ng/ml, median (IQR) & $243.9(74.9-790.8)$ \\
Hb, g/dL, median (IQR) & $12.1(10.3-13.5)$ \\
Ca, g/dL, median (IQR) & $8.7(8.3-9.1)$ \\
ALP, U/L, median (IQR) & $118(75-227)$ \\
CRPC, $n$ (\%) & \\
Yes & $375(57.9 \%)$ \\
No & $273(42.1 \%)$ \\
PSA kinetics after ADT & \\
TTN, month, median (IQR) & $8.7(4.8-16.65)$ \\
Nadir PSA level, ng/ml, median (IQR) & $0.86(0.07-5.99)$ \\
PSARR, \%/month, median (IQR) & $10.32(5.62-18.17)$ \\
TFNTC, month, median (IQR) & $4.5(2.7-15.1)$ \\
PSADT, month, median (IQR) & $2.6(1.5-5.3)$ \\
\hline CRPC: catration-resistat proste cancr & \\
\hline
\end{tabular}

CRPC: castration-resistant prostate cancer, TTN: time to PSA nadir, PSARR: PSA reduction rate, TFNTC: time from PSA nadir to CRPC, and PSADT: PSA doubling time.

groups based on tumor volume, subsequently demonstrating a statistically significant improvement in OS for patients with HVD on chemohormonal therapy [27].

HVD accounted for $54 \%$ of our study population, $63 \%$ of that in the CHARTEED trial, and $49 \%$ of that in previous studies within Taiwan [20]. The present study found that patients on ADT with HVD had significantly shorter OS (30 vs. 43 months) and time to the development of CRPC (13 vs. 26 months) compared to those with LVD. This agrees with the general consensus that HVD promotes worse prognosis due to disease severity.

PSA level has been the most widely used biomarker for evaluating disease progression and predicting survival in clinical practice. Furthermore, several retrospective clinical studies and even some meta-analyses have determined that PSA kinetics, including PSA response rate, nadir PSA level, TTN, or PSADT, predicted OS or disease progression $[9,10,13,24]$. PSADT predicting outcomes has been known for nearly 30 years, and it may closely indicate changes in prostate tumor volume, an independent predictor of biochemical relapse among patients that either underwent radical prostatectomy or endocrine treatment [28]. Doctor D'Amico et al. demonstrated that patients with clinically localized prostate cancer treated with external beam radiation therapy with PSADT $>12$ months have lower risk of prostate cancer death within 5 years of relapse [29]. Moreover, Kelloff et al. reported that PSADT was a predictor of tumor response to medication in patients with prostate cancer and suggested that significant changes in PSADT may be used to support the approval of newer treatment [30]. Despite the considerably wide distribution of PSADT values, studies have suggested that it may still be useful for strategies after relapse and that patients with advanced or relapsed disease who have rapid PSADT should receive more aggressive or earlier treatment [31, 32]. Tomioka et al. also reported that posttreatment PSADT $\leq 2$ months may be a predictor for decreased survival and was associated with poor prognosis among patients with bone metastatic prostate cancer [33]. The present study identified lower cutoff point of PSADT than before as a useful clinical predictor in patients with LVD receiving ADT (PSADT $<4$ months). The possible cause of short median PSADT in our study is that the PSA nadir level is low, so that the doubled PSA level could be reached easily. In addition, PSA level is checked more frequently in our study than in other studies, so the PSADT may also be influenced. Although PSADT cannot be evaluated during the early stages of ADT, it may still be a clinically effective predictor of disease progression and OS.

Previous reports have shown that nadir PSA levels, TTN, and even PSA reduction pattern have been considered important predictors of survival and progression period. Generally, a faster decline in PSA levels has been associated with more cancer cell death, which promotes a more favourable prognosis and survival [34]. However, Ji et al. reported in their retrospective study that rapidly decreasing PSA levels during initial ADT was a risk factor for early progression to CRPC [16]. Accordingly, a recent study introduced the novel concept suggesting that tumor-regulating fibroblasts play an important role in the mechanisms associated with TTN after primary ADT [35]. Other studies have also demonstrated that rapidly decreasing PSA levels may be associated with transcriptional outcomes of ADT rather than cancer cell death. Moreover, heterogeneous prostate cancer cells, including hormone-resistant prostate cancer cells and hormone-sensitive prostate cancer cells, often coexist in the same patient. The rapid decline in PSA levels may indicate downregulation of PSA expression in hormone-sensitive prostate cancer cells, which are regulated by androgen via the androgen receptor pathway [36]. Although it is doubtful that whether hormone-sensitive prostate cancer cells account for more of the cancer cells in HVD than in LVD, it might be a possible explanation for patient with HVD to have shorter TTN and faster time to disease progression, even shorter OS. Moreover, longer TTN and lower PSA nadir levels during ADT have been known to be associated with significantly longer OS and period of disease progression $[10,11,13,24,37]$. Hamano et al. demonstrated that higher PSA nadir and shorter TTN are poor prognostic factors in men with MCRPC on ADT [24], and we might presume that TTN and PSA nadir are the effective predictors for disease progression in relative advanced prostate cancer. Given that LVD has been associated with a lower CRPC rate and less severe bone metastases and disease status, TTN, PSA nadir, and PSA decline pattern might not be effective predictors for OS and disease 


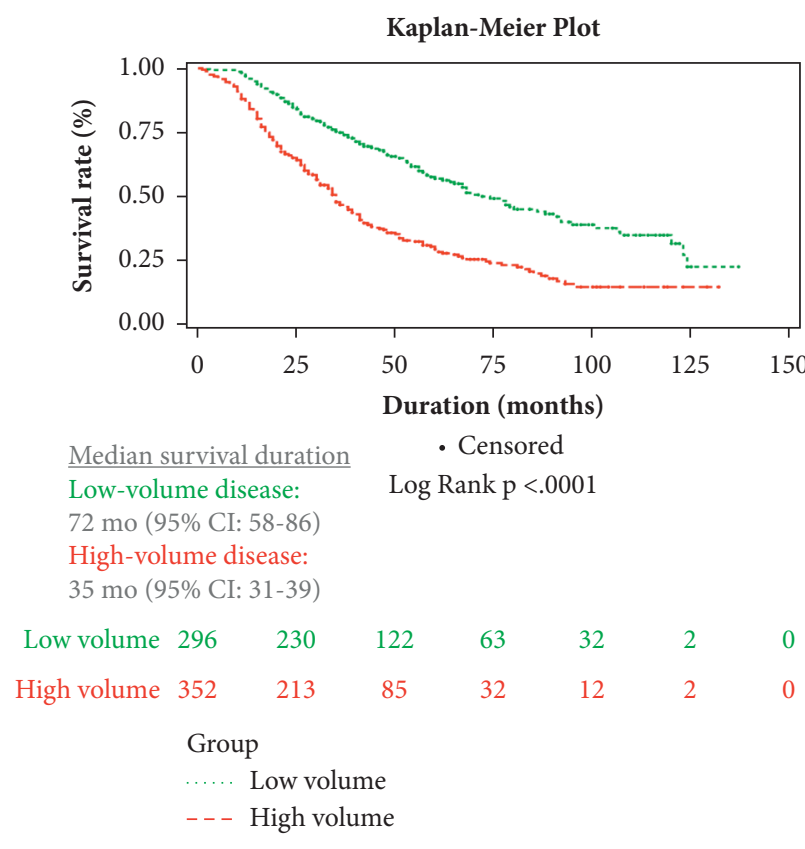

(a)

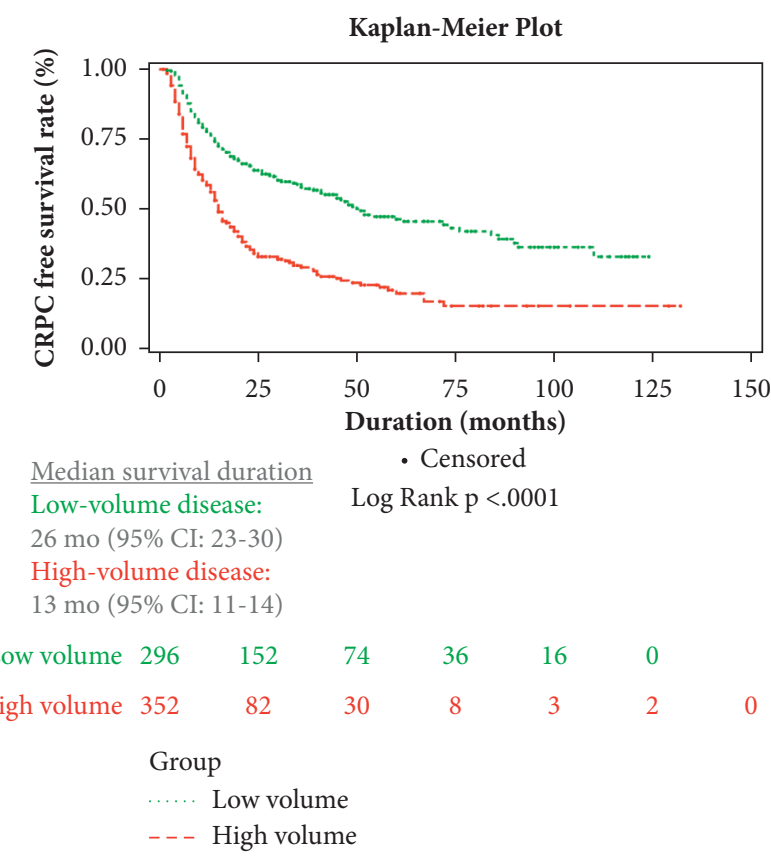

(b)

FIgURE 1: (a) Kaplan-Meier plot of overall survival with high-volume disease and low-volume disease. (b) Kaplan-Meier plot of CRPC free survival between patients with high-volume disease and low-volume disease. CI: confidence interval.

TABLE 3: PSA kinetics after ADT in LVD.

\begin{tabular}{|c|c|c|c|}
\hline & CRPC $(143,48.3 \%)$ & Non-CRPC $(153,51.7 \%)$ & \\
\hline TTN, month, median (IQR) & $8.7(5.4-14.1)$ & $16.7(8.7-26.6)$ & $<0.0001$ \\
\hline Nadir PSA level, ng/ml, median (IQR) & $0.75(0.12-2.92)$ & $0.04(0.01-0.41)$ & 0.618 \\
\hline 3-month PSARR, \%, median (IQR) & $95.64(87.67-98.54)$ & $97.18(92.65-99.16)$ & 0.214 \\
\hline TFNTC, month, median (IQR) & $4.3(2.7-11.65)$ & $15.6(4-41.1)$ & $<0.0001$ \\
\hline PSADT, month, median (IQR) & $3.65(1.9-6.875)$ & $3.4(1.95-7.325)$ & 0.534 \\
\hline
\end{tabular}

CRPC: castration-resistant prostate cancer, TTN: time to PSA nadir, 3-month PSARR: 3-month PSA reduction rate, TFNTC: time from PSA nadir to CRPC, and PSADT: PSA doubling time.

progression in LVD. Notably, PSA response rates (even 4 weeks, 12 weeks, or maximal response rate) were used to evaluate the treatment response for patients with prostate cancer in previous studies $[38,39]$, so in LVD, even though PSA decline rate is not a major predictor for OS or disease progression in the present study, it might still have the role in treatment response evaluation.

The major implication of the current study is that, among patients with LVD, more attention should be provided to those with increasing PSA levels and PSADT considering that TTN, PSA nadir, and even PSA reduction pattern were not major predictors for faster disease progression or OS. To our knowledge, early chemotherapy or androgen metabolism inhibitors (abiraterone with prednisone) therapy is not recommended in LVD initially, but some of them might be suitable for early usage of second-generation antiandrogen such as apalutamide and enzalutamide. In the present study, disease that progressed to CRPC was noted in $143(48.3 \%)$ patients with LVD, and we found that they had similar nadir PSA level, 3-month PSA reduction rate, and PSADT, but significantly faster TTN (8.7 vs. 16.7 months) and faster TFNTC (4.3 vs. 15.6 months) $(p<0.001)$ compared to patients with no CRPC in LVD. In addition, in univariate analysis, TTN and TFNTC were also statistically significant parameters for disease progression. Even though in multivariate analysis, only PSADT is shown to be predictor for disease progression, we can still hypothesize that patient with faster TTN and shorter TFNTC might be related to disease that progressed to CRPC in LVD. The possible cause is that TTN and TFNTC are significantly different in patient with CRPC or not, so the cutoff point we chose might not be the optimal cutoff point for analysis. Even though TTN and TFNTC are not the major predictors for disease progression in LVD, they might still be the parameters for disease control evaluation. Patients with faster TTN and shorter TFNTC might be the hints, even the predictors for poor disease control status for us so that we could early apply chemotherapy or the new generation hormone therapy such as abiraterone or enzalutamide for better disease control or quality of life improvement. 
TABLE 4: Univariate and multivariate analysis of predicting factors for time to CRPC.

\begin{tabular}{|c|c|c|c|c|}
\hline Factors & Univariate hazard ratio $(95 \% \mathrm{CI})$ & $p$ value & Multivariate hazard ratio (95\% CI) & $p$ value \\
\hline \multicolumn{5}{|l|}{ Age } \\
\hline$<75$ & 1 (reference) & & & \\
\hline$\geq 75$ & $0.97(0.79-1.18)$ & 0.731 & - & \\
\hline \multicolumn{5}{|c|}{ Gleason grade group } \\
\hline$<4$ & 1 (reference) & & & \\
\hline$\geq 4$ & $1.75(1.25-2.46)$ & 0.001 & - & \\
\hline \multicolumn{5}{|c|}{ Initial PSA level, $\mathrm{ng} / \mathrm{ml}$} \\
\hline$<250$ & 1 (reference) & & & \\
\hline$\geq 250$ & $1.69(1.34-2.14)$ & $<0.0001$ & - & \\
\hline \multicolumn{5}{|c|}{ TTN, month } \\
\hline$<9$ & 1 (reference) & & 1 (reference) & \\
\hline$\geq 9$ & $0.27(0.22-0.33)$ & $<0.0001$ & $0.22(0.11-0.44)$ & $<0.0001$ \\
\hline \multicolumn{5}{|c|}{ Nadir PSA level, ng/ml } \\
\hline$<1$ & 1 (reference) & & 1 (reference) & \\
\hline$\geq 1$ & $3.78(2.93-4.88)$ & $<0.0001$ & $3.11(2.25-4.29)$ & $<0.0001$ \\
\hline \multicolumn{5}{|c|}{ PSARR, \% (month) } \\
\hline$<10$ & 1 (reference) & & 1 (reference) & \\
\hline$\geq 10$ & $3.76(2.93-4.81)$ & $<0.0001$ & $0.59(0.31-1.15)$ & 0.123 \\
\hline \multicolumn{5}{|c|}{ TFNTC, month } \\
\hline$<5$ & 1 (reference) & & 1 (reference) & \\
\hline$\geq 5$ & $0.19(0.15-0.24)$ & $<0.0001$ & $1.37(0.99-1.89)$ & 0.059 \\
\hline \multicolumn{5}{|c|}{ PSADT, month } \\
\hline$<3$ & 1 (reference) & & 1 (reference) & \\
\hline$\geq 3$ & $0.34(0.26-0.44)$ & $<0.0001$ & $0.34(0.25-0.48)$ & $<0.0001$ \\
\hline
\end{tabular}

TTN: time to PSA nadir, PSARR: PSA reduction rate, TFNTC: time from PSA nadir to CRPC, and PSADT: PSA doubling time.

TABLE 5: Univariate and multivariate analysis of predicting factors for OS.

\begin{tabular}{|c|c|c|c|c|}
\hline Factors & Univariate hazard ratio $(95 \% \mathrm{CI})$ & $p$ value & Multivariate hazard ratio $(95 \% \mathrm{CI})$ & $p$ value \\
\hline \multicolumn{5}{|l|}{ Age } \\
\hline$<75$ & 1 (reference) & & & \\
\hline$\geq 75$ & $1.37(0.11-1.68)$ & $<0.001$ & - & \\
\hline \multicolumn{5}{|c|}{ Gleason grade group } \\
\hline$<4$ & 1 (reference) & & & \\
\hline$\geq 4$ & $1.82(1.29-2.59)$ & $<0.0001$ & - & \\
\hline \multicolumn{5}{|c|}{ Initial PSA level, $\mathrm{ng} / \mathrm{ml}$} \\
\hline$<250$ & 1 (reference) & & & \\
\hline$\geq 250$ & $1.29(1.03-1.62)$ & 0.030 & - & \\
\hline \multicolumn{5}{|c|}{ TTN, month } \\
\hline$<9$ & 1 (reference) & & 1 (reference) & \\
\hline$\geq 9$ & $0.25(0.20-0.31)$ & $<0.0001$ & $0.24(0.12-0.48)$ & $<0.0001$ \\
\hline \multicolumn{5}{|c|}{ Nadir PSA level, ng/ml } \\
\hline$<1$ & 1 (reference) & & 1 (reference) & \\
\hline$\geq 1$ & $3.66(2.86-4.68)$ & $<0.0001$ & $2.76(1.98-3.84)$ & $<0.0001$ \\
\hline \multicolumn{5}{|c|}{ PSARR, \% (month) } \\
\hline$<10$ & 1 (reference) & & 1 (reference) & \\
\hline$\geq 10$ & $3.51(2.73-4.51)$ & $<0.0001$ & $0.53(0.27-1.04)$ & 0.065 \\
\hline \multicolumn{5}{|c|}{ TFNTC, month } \\
\hline$<5$ & 1 (reference) & & 1 (reference) & \\
\hline$\geq 5$ & $0.38(0.30-0.47)$ & $<0.0001$ & $1.67(1.18-2.37)$ & 0.004 \\
\hline \multicolumn{5}{|c|}{ PSADT, month } \\
\hline$<3$ & 1 (reference) & & 1 (reference) & \\
\hline$\geq 3$ & $0.25(0.19-0.34)$ & $<0.0001$ & $0.40(0.28-0.56)$ & $<0.0001$ \\
\hline \multicolumn{5}{|c|}{ TTC, month } \\
\hline$<17$ & 1 (reference) & & 1 (reference) & \\
\hline$\geq 17$ & $0.20(0.16-0.25)$ & $<0.0001$ & $0.53(0.32-0.85)$ & 0.009 \\
\hline
\end{tabular}

TTN: time to PSA nadir, PSARR: PSA reduction rate, TFNTC: time from PSA nadir to CRPC, PSADT: PSA doubling time, and TTC: time from ADT to CRPC. 
TABLE 6: Univariate and multivariate analysis of predicting factors for time to CRPC in LVD.

\begin{tabular}{|c|c|c|c|c|}
\hline Factors & Univariate hazard ratio $(95 \% \mathrm{CI})$ & $p$ value & Multivariate hazard ratio $(95 \% \mathrm{CI})$ & $p$ value \\
\hline \multicolumn{5}{|l|}{ Age } \\
\hline$<75$ & 1 (reference) & & & \\
\hline$\geq 75$ & $0.94(0.67-1.30)$ & 0.699 & - & \\
\hline \multicolumn{5}{|c|}{ Gleason grade group } \\
\hline$<4$ & 1 (reference) & & & \\
\hline$\geq 4$ & $1.82(1.13-2.96)$ & 0.015 & - & \\
\hline \multicolumn{5}{|c|}{ Initial PSA level, $\mathrm{ng} / \mathrm{ml}$} \\
\hline$<100$ & 1 (reference) & & & \\
\hline$\geq 100$ & $1.03(0.71-1.51)$ & 0.864 & - & \\
\hline \multicolumn{5}{|l|}{ TTN, month } \\
\hline$<12$ & 1 (reference) & & 1 (reference) & \\
\hline$\geq 12$ & $0.25(0.18-0.36)$ & $<0.0001$ & $0.31(0.05-2.01)$ & 0.222 \\
\hline \multicolumn{5}{|c|}{ Nadir PSA level, ng/ml } \\
\hline$<0.2$ & 1 (reference) & & 1 (reference) & \\
\hline$\geq 0.2$ & $5.61(3.62-8.69)$ & $<0.0001$ & $2.09(0.88-4.94)$ & 0.094 \\
\hline \multicolumn{5}{|c|}{ 3-mon PSARR, \% } \\
\hline \multicolumn{5}{|c|}{$<97$} \\
\hline$\geq 97$ & 1 (reference) & & & \\
\hline $1.49(0.98-2.26)$ & 0.063 & & & \\
\hline \multicolumn{5}{|c|}{ PSARR, \% (month) } \\
\hline$<8$ & 1 (reference) & & 1 (reference) & \\
\hline$\geq 8$ & $4.20(2.79-6.35)$ & $<0.0001$ & $0.79(0.13-4.86)$ & 0.802 \\
\hline \multicolumn{5}{|l|}{ TFNTC, month } \\
\hline$<8$ & 1 (reference) & & 1 (reference) & \\
\hline$\geq 8$ & $0.18(0.12-0.26)$ & $<0.0001$ & $0.97(0.38-2.46)$ & 0.944 \\
\hline \multicolumn{5}{|l|}{ PSADT, month } \\
\hline$<4$ & 1 (reference) & & 1 (reference) & \\
\hline$\geq 4$ & $0.45(0.30-0.68)$ & $<0.0001$ & $0.31(0.16-0.61)$ & 0.001 \\
\hline
\end{tabular}

TTN: time to PSA nadir, 3-mon PSARR: posttreatment 3 months' PSA reduction rate, PSARR: PSA reduction rate, TFNTC: time from PSA nadir to CRPC, and PSADT: PSA doubling time.

TABLE 7: Univariate and multivariate analysis of predicting factors for OS in LVD.

\begin{tabular}{|c|c|c|c|c|}
\hline Factors & Univariate hazard ratio $(95 \% \mathrm{CI})$ & $p$ value & Multivariate hazard ratio $(95 \% \mathrm{CI})$ & $p$ value \\
\hline \multicolumn{5}{|l|}{ Age } \\
\hline$<75$ & 1 (reference) & & & \\
\hline$\geq 75$ & $1.64(1.15-2.32)$ & 0.006 & - & \\
\hline \multicolumn{5}{|c|}{ Gleason grade group } \\
\hline$<4$ & 1 (reference) & & & \\
\hline$\geq 4$ & $1.71(1.04-2.82)$ & 0.034 & - & \\
\hline \multicolumn{5}{|c|}{ Initial PSA level, ng/ml } \\
\hline$<100$ & 1 (reference) & & & \\
\hline$\geq 100$ & $1.000 .68-1.48)$ & 0.995 & - & \\
\hline \multicolumn{5}{|c|}{ TTN, month } \\
\hline$<12$ & 1 (reference) & & 1 (reference) & \\
\hline$\geq 12$ & $0.30(0.21-0.44)$ & $<0.0001$ & $0.32(0.05-1.93)$ & 0.214 \\
\hline \multicolumn{5}{|c|}{ Nadir PSA level, ng/ml } \\
\hline$<0.2$ & 1 (reference) & & 1 (reference) & \\
\hline$\geq 0.2$ & $3.37(2.20-5.17)$ & $<0.0001$ & $1.87(0.78-4.49)$ & 0.159 \\
\hline \multicolumn{5}{|c|}{ 3-mon PSARR, \% } \\
\hline$<97$ & 1 & 0.108 & & \\
\hline$\geq 97$ & $1.44(0.92-2.24)$ & & & \\
\hline \multicolumn{5}{|c|}{ PSARR, \% (month) } \\
\hline$<8$ & 1 (reference) & & 1 (reference) & \\
\hline$\geq 8$ & $3.99(2.58-6.19)$ & $<0.0001$ & $0.71(0.12-4.13)$ & 0.703 \\
\hline \multicolumn{5}{|c|}{ TFNTC, month } \\
\hline$<8$ & 1 (reference) & & 1 (reference) & \\
\hline$\geq 8$ & $0.28(0.19-0.40)$ & $<0.0001$ & $1.21(0.46-3.19)$ & 0.706 \\
\hline
\end{tabular}


TABLE 7: Continued.

\begin{tabular}{lccc}
\hline Factors & Univariate hazard ratio (95\% CI) & $p$ value & Multivariate hazard ratio (95\% CI) \\
\hline PSADT, month & 1 (reference) & & 1 (reference) \\
$<4$ & $0.19(0.11-0.32)$ & $<0.0001$ & $0.35(0.17-0.71)$ \\
$\geq 4$ & 1 (reference) & & \\
TTC, month & $0.18(0.12-0.26)$ & $<0.0001$ & 1 (reference) \\
$<26$ & 26 & $0.57(0.20-1.64)$ & 0.004 \\
\hline
\end{tabular}

TTN: time to PSA nadir, 3-mon PSARR: posttreatment 3 months' PSA reduction rate, PSARR: PSA reduction rate, TFNTC: time from PSA nadir to CRPC, PSADT: PSA doubling time, and TTC: time from ADT to CRPC.

\section{Limitations}

The current study has some limitations mentioned as follows. First, the most important limitation is our exclusion of patients who received chemotherapy or radiotherapy; however, those with subsequent hormone therapy, including abiraterone or enzalutamide after CRPC, were not excluded, which may have affected our results with regard to OS. Second, given that this was a single-centre retrospective study, our results may not be generalisable to other populations. Moreover, considering that Linkou CGMH is a medical centre in northern Taiwan, the disease severities of our study population may be higher than those of general population. Lastly, not all PSA parameters and factors, including Gleason score, $\mathrm{Hb}, \mathrm{Ca}$, and ALP, were regularly assessed at our institution, which might have influenced our results.

\section{Conclusion}

Among patients receiving ADT, those with high-volume metastatic prostate cancer had significantly shorter OS and faster disease progression compared to those with lowvolume metastatic prostate cancer. Moreover, the current study demonstrated that PSADT $\geq 4$ months had been identified as an effective predictor for slower disease progression and better OS in LVD. Furthermore, other PSA kinetics including TTN, PSA decline pattern, and PSA nadir were not the major predictors for disease progression or OS in LVD, but TTN and time from PSA nadir to CRPC (TFNTC) might be the predictors for disease control status.

\section{Abbreviations}

mPCa: Metastatic prostate cancer

ADT: Androgen deprivation therapy

CRPC: Castration-resistant prostate cancer

OS: Overall survival

HVD: $\quad$ High-volume disease

PSA: $\quad$ Prostate-specific antigen

iPSA: Initial PSA level

TTN: $\quad$ Time to PSA nadir

PSADT: PSA doubling time

$\mathrm{Hb}$ : Haemoglobin

ALP: $\quad$ Alkaline phosphatase

mCNPC: Metastatic castration-naïve prostate cancer

mCRPC: Metastatic castration-resistant prostate cancer

LVD: Low-volume disease

Ca: $\quad$ Calcium
PSARR: PSA reduction rate

TFNTC: Time from PSA nadir to CRPC.

\section{Data Availability}

This study is based in part on data from the Chang Gung Research Database provided by Chang Gung Memorial Hospital. The interpretation and conclusions contained herein do not represent the position of Chang Gung Memorial Hospital.

\section{Ethical Approval}

This study was approved by the Institutional Review Board (IRB) of Chang Gung Medical Foundation (IRB number 201801377B0).

\section{Consent}

Patient consent is not required for observational studies.

\section{Disclosure}

The manuscript's preprint can be found in the following link: https://www.researchsquare.com/article/rs-117622/v1.

\section{Conflicts of Interest}

The authors declare that they have no conflicts of interest.

\section{Acknowledgments}

The authors wish to acknowledge the Maintenance Project of the Center for Big Data Analytics and Statistics (Grant no. CLRPG3D0046) at Chang Gung Memorial Hospital for their support in the study design and monitoring, data analysis, and interpretation. The present study was supported by grants from The Chang Gung Memorial Hospital Research Foundation (grant nos. CMRPG3F0701-2 and CORPG3F0271; Taoyuan, Taiwan).

\section{References}

[1] D. C. Grossman, D. C. Grossman, S. J. Curry et al., "Screening for prostate cancer," JAMA, vol. 319, no. 18, pp. 1901-1913, 2018.

[2] C.-F. Hung, C.-K. Yang, and Y.-C. Ou, "Urologic cancer in taiwan," Japanese Journal of Clinical Oncology, vol. 46, no. 7, pp. 605-609, 2016. 
[3] Health Promotion Administration MoHaW, Cancer Registry Annual Report, 2017, Taiwan, Taiwan: Health Promotion Administration, Ministry of Health and Welfare, Taipei, Taiwan, 2019.

[4] J. Ferlay, I. Soerjomataram, R. Dikshit et al., "Cancer incidence and mortality worldwide: sources, methods and major patterns in GLOBOCAN 2012," International Journal of Cancer, vol. 136, no. 5, pp. E359-E386, 2015.

[5] C. J. Sweeney, Y.-H. Chen, M. Carducci et al., "Chemohormonal therapy in metastatic hormone-sensitive prostate cancer," New England Journal of Medicine, vol. 373, no. 8, pp. 737-746, 2015.

[6] W.-A. Kwon, J. Y. Joung, J. E. Lee et al., "Use of docetaxel plus androgen deprivation therapy for metastatic hormone-sensitive prostate cancer in Korean patients: a retrospective study," Investigative and Clinical Urology, vol. 60, no. 3, pp. 195-201, 2019.

[7] N. D. James, M. R. Sydes, N. W. Clarke et al., "Addition of docetaxel, zoledronic acid, or both to first-line long-term hormone therapy in prostate cancer (STAMPEDE): survival results from an adaptive, multiarm, multistage, platform randomised controlled trial," The Lancet, vol. 387, no. 10024, pp. 1163-1177, 2016.

[8] G. Gravis, K. Fizazi, F. Joly et al., “Androgen-deprivation therapy alone or with docetaxel in non-castrate metastatic prostate cancer (GETUG-AFU 15): a randomised, open-label, phase 3 trial," The Lancet Oncology, vol. 14, no. 2, pp. 149-158, 2013.

[9] A. Afriansyah, A. R. A. H. Hamid, C. A. Mochtar, and R. Umbas, "Prostate specific antigen (PSA) kinetic as a prognostic factor in metastatic prostate cancer receiving androgen deprivation therapy: systematic review and metaanalysis," F1000Research, vol. 7, p. 246, 2018.

[10] J. Y. C. Teoh, J. H. L. Tsu, S. K. K. Yuen et al., "Prognostic significance of time to prostate-specific antigen (PSA) nadir and its relationship to survival beyond time to PSA nadir for prostate cancer patients with bone metastases after primary androgen deprivation therapy," Annals of Surgical Oncology, vol. 22, no. 4, pp. 1385-1391, 2015.

[11] S.-P. Huang, B.-Y. Bao, M.-T. Wu et al., "Impact of prostatespecific antigen (PSA) nadir and time to PSA nadir on disease progression in prostate cancer treated with androgen-deprivation therapy," The Prostate, vol. 71, no. 11, pp. 1189-1197, 2011.

[12] T. K. Choueiri, W. Xie, A. V. D'amico et al., “Time to prostatespecific antigen nadir independently predicts overall survival in patients who have metastatic hormone-sensitive prostate cancer treated with androgen-deprivation therapy," Cancer, vol. 115, no. 5, pp. 981-987, 2009.

[13] A. Tomioka, N. Tanaka, M. Yoshikawa et al., "Nadir PSA level and time to nadir PSA are prognostic factors in patients with metastatic prostate cancer," BMC Urology, vol. 14, no. 1, p. 33, 2014.

[14] A. L. Zietman, M. K. Tibbs, K. C. Dallow et al., "Use of PSA nadir to predict subsequent biochemical outcome following external beam radiation therapy for T1-2 adenocarcinoma of the prostate," Radiotherapy \& Oncology, vol. 40, no. 2, pp. 159-162, 1996.

[15] E. Akbay, M. Bozlu, S. Çayan, P. Ö. Kara, M. Tek, and C. Aytekin, "Prostate-specific antigen decline pattern in advanced prostate cancer receiving androgen deprivation therapy and relationship with prostate-specific antigen progression," The Aging Male: The Official Journal of the
International Society for the Study of the Aging Male, vol. 20, no. 3, pp. 175-183, 2017.

[16] G. Ji, G. Song, C. Huang, S. He, and L. Zhou, "Rapidly decreasing level of prostate-specific antigen during initial androgen deprivation therapy is a risk factor for early progression to castration-resistant prostate cancer: a retrospective study," Medicine, vol. 96, no. 36, p. e7823, 2017.

[17] K. C. Koo, S. U. Park, K. H. Kim et al., "Predictors of survival in prostate cancer patients with bone metastasis and extremely high prostate-specific antigen levels," Prostate International, vol. 3, no. 1, pp. 10-15, 2015.

[18] R. C. Semeniuk, P. M. Venner, and S. North, "Prostate-specific antigen doubling time is associated with survival in men with hormone-refractory prostate cancer," Urology, vol. 68, no. 3, pp. 565-569, 2006.

[19] N. Terada, S. Akamatsu, T. Kobayashi, T. Inoue, O. Ogawa, and E. S. Antonarakis, "Prognostic and predictive biomarkers in prostate cancer: latest evidence and clinical implications," Therapeutic Advances in Medical Oncology, vol. 9, no. 8, pp. 565-573, 2017.

[20] C.-H. Chen, Y.-T. Cheng, J.-H. Hong et al., "Impact of highvolume disease in Asian population with newly diagnosed metastatic prostate cancer," Urological Science, vol. 29, no. 3, p. 136, 2018.

[21] B. Delahunt, L. Egevad, J. R. Srigley et al., "Validation of International Society of Urological Pathology (ISUP) grading for prostatic adenocarcinoma in thin core biopsies using TROG 03.04 "RADAR" trial clinical data," Pathology, vol. 47, no. 6, pp. 520-525, 2015.

[22] T.-T. Lin, Y.-H. Chen, Y.-P. Wu et al., "Risk factors for progression to castration-resistant prostate cancer in metastatic prostate cancer patients," Journal of Cancer, vol. 10, no. 22 , pp. 5608-5613, 2019.

[23] S. Tamada, T. Iguchi, M. Kato et al., "Time to progression to castration-resistant prostate cancer after commencing combined androgen blockade for advanced hormone-sensitive prostate cancer," Oncotarget, vol. 9, no. 97, pp. 36966-36974, 2018.

[24] I. Hamano, S. Hatakeyama, S. Narita et al., "Impact of nadir PSA level and time to nadir during initial androgen deprivation therapy on prognosis in patients with metastatic castration-resistant prostate cancer," World Journal of Urology, vol. 37, no. 11, pp. 2365-2373, 2019.

[25] C. A. Whitney, L. E. Howard, S. J. Freedland et al., "Impact of age, comorbidity, and PSA doubling time on long-term competing risks for mortality among men with non-metastatic castration-resistant prostate cancer," Prostate Cancer and Prostatic Diseases, vol. 22, no. 2, pp. 252-260, 2019.

[26] M. Nakayama, H. Kobayashi, T. Takahara, R. Oyama, K. Imanaka, and K. Yoshizawa, "Association of early PSA decline and time to PSA progression in abiraterone acetatetreated metastatic castration-resistant prostate cancer; a posthoc analysis of Japanese phase 2 trials," BMC Urology, vol. 16, no. 1, p. 27, 2016.

[27] S. Damodaran, C. E. Kyriakopoulos, and D. F. Jarrard, "Newly diagnosed metastatic prostate cancer: has the paradigm changed?" Urologic Clinics of North America, vol. 44, no. 4, pp. 611-621, 2017.

[28] G. Tisman, "Describing prostate cancer dynamics: second look at PSA-doubling time and PSA-specific growth rate," in Advances in Prostate Cancer, vol. 177, Springer, Germany, 2013.

[29] A. V. D’Amico, K. Cote, M. Loffredo, A. A. Renshaw, and D. Schultz, "Determinants of prostate cancer-specific survival 
after radiation therapy for patients with clinically localized prostate cancer," Journal of Clinical Oncology, vol. 20, no. 23, pp. 4567-4573, 2002.

[30] G. J. Kelloff, D. S. Coffey, B. A. Chabner et al., "Prostatespecific antigen doubling time as a surrogate marker for evaluation of oncologic drugs to treat prostate cancer," Clinical Cancer Research, vol. 10, no. 11, pp. 3927-3933, 2004.

[31] A. J. Vickers and S. F. Brewster, "PSA velocity and doubling time in diagnosis and prognosis of prostate cancer," British Journal of Medical and Surgical Urology, vol. 5, no. 4, pp. 162-168, 2012.

[32] S. Nakata, H. Takahashi, Y. Takezawa et al., "PSA doubling time in prostate cancer relapsed after endocrine therapy," The Japanese Journal of Urology, vol. 91, no. 7-8, pp. 584-588, 2000.

[33] S. Tomioka, M. Shimbo, Y. Amiya, H. Nakatsu, S. Murakami, and J. Shimazaki, "Significance of prostate-specific antigendoubling time on survival of patients with hormone refractory prostate cancer and bone metastasis: analysis on 56 cases of cancer-specific death," International Journal of Urology, vol. 14, no. 2, pp. 123-127, 2007.

[34] Y. Arai, T. Yoshiki, and O. Yoshida, "Prognostic significance of prostate specific antigen in endocrine treatment for prostatic cancer," The Journal of Urology, vol. 144, no. 6, pp. 1415-1419, 1990.

[35] T. Sasaki and Y. Sugimura, "The importance of time to prostate-specific antigen (PSA) nadir after primary androgen deprivation therapy in hormone-naïve prostate cancer patients," Journal of Clinical Medicine, vol. 7, no. 12, p. 565, 2018.

[36] T. Sasaki, T. Onishi, and A. Hoshina, "Nadir PSA level and time to PSA nadir following primary androgen deprivation therapy are the early survival predictors for prostate cancer patients with bone metastasis," Prostate Cancer and Prostatic Diseases, vol. 14, no. 3, pp. 248-252, 2011.

[37] T. Sasaki, T. Onishi, and A. Hoshina, "Cutoff value of time to prostate-specific antigen nadir is inversely correlated with disease progression in advanced prostate cancer," EndocrineRelated Cancer, vol. 19, no. 5, pp. 725-730, 2012.

[38] D. E. Rathkopf and H. I. Scher, "Apalutamide for the treatment of prostate cancer," Expert Review of Anticancer Therapy, vol. 18, no. 9, pp. 823-836, 2018.

[39] P. Rescigno, D. Lorente, D. Bianchini et al., "Prostate-specific antigen decline after 4 weeks of treatment with abiraterone acetate and overall survival in patients with metastatic castration-resistant prostate cancer," European Urology, vol. 70, no. 5, pp. 724-731, 2016. 